\title{
Romain Rolland et le théâtre populaire suisse
}

Une rencontre dramaturgique

\section{Alain Corbellari}

\section{(2) OpenEdition}

\section{Journals}

Édition électronique

URL : http://journals.openedition.org/edl/349

DOI : 10.4000/edl.349

ISSN : 2296-5084

Éditeur

Université de Lausanne

\section{Édition imprimée}

Date de publication : 15 septembre 2012

Pagination : 105-118

ISBN : 978-2-940331-28-4

ISSN : 0014-2026

\section{Référence électronique}

Alain Corbellari, «Romain Rolland et le théâtre populaire suisse », Études de lettres [En ligne], 3 | 2012, mis en ligne le 15 septembre 2015, consulté le 22 décembre 2020. URL : http:// journals.openedition.org/edl/349; DOI : https://doi.org/10.4000/edl.349 


\section{ROMAIN ROLLAND ET LE THÉÂTRE POPULAIRE SUISSE: UNE RENCONTRE DRAMATURGIQUE}

Le renouvellement de la dramaturgie moderne a été une des préoccupations constantes de Romain Rolland. Proche de Maurice Pottecher et de son «Théâtre du peuple», expérimenté à Bussang dès 1899, Rolland a trouvé dans les spectacles populaires vus en Suisse dans la première décennie du XXe siècle un stimulant puissant à son imagination théâtrale. Entre la nostalgie rousseauiste d'un théâtre dont nul n'est exclu et l'utopie d'un théâtre en liberté anticipant sur les tentatives radicales du second aprèsguerre, Romain Rolland aura trouvé dans les traditions suisses une raison de plus de se sentir proche du pays qui a véritablement été sa seconde patrie.

Romain Rolland n'a pas attendu d'y trouver un refuge durant la Première Guerre mondiale pour découvrir la Suisse. Très tôt le territoire helvétique lui a paru le moyen terme idéal entre une Allemagne rêvée où il ne mettra, significativement, presque jamais les pieds et une Italie passionnément aimée dès son séjour d'études romain de 1889 à 1891, au cours duquel il rencontra Malwida von Meysenbug, fameuse admiratrice de Wagner et de Nietzsche. Rolland fera ainsi des voyages réguliers en Suisse dès sa vingtième année et élira d'emblée les bords du Léman comme étape privilégiée de ses pérégrinations. L’y attachent en particulier le souvenir de Rousseau, dont la dénonciation des méfaits de la civilisation est si proche de la sienne ${ }^{1}$, mais aussi celui de... Victor Hugo qu'il avait en effet entr'aperçu à l'hôtel Byron de Villeneuve en 1883,

I. Malgré ou peut-être à cause des nombreux points de rencontre avec sa pensée, Rolland porta toujours des jugements assez ambivalents sur Rousseau. Voir A. Corbellari, Les mots sous les notes, p. 52-54. 
alors qu'il avait 17 ans $^{2}$. On sait d'ailleurs que c'est dans la proximité immédiate de cet hôtel mythique, à la "villa Olga», qu'il s'installera, de 1922 à 1938, face à un paysage dont la majesté lui apparaissait propice à son travail biographico-musicologique sur Beethoven, comme en témoigne une lettre écrite de Vézelay à Charles Baudouin, peu après son déménagement:

On ne peut imaginer harmonies plus différentes que celle de la villa de Villeneuve et celle de la petite maison de Vézelay. Mais elles se valent. L'une est Beethoven, l'autre Mozart: (les comparaisons ne valent rien!). Ce n'est plus l'assaut vers les cimes. On plane ici sur l'étendue 3 .

Les rapports de Rolland avec les musicologues suisses mériteraient assurément une étude approfondie, car elles restent assez mal connues: entre 1900 et 1912, alors que la musicologie représente l'activité essentielle de notre auteur, la Suisse semble cependant surtout rester pour lui un séjour de vacances; mais lorsque Rolland aura enfin l'occasion, aprèsguerre, de nouer des contacts plus approfondis avec les ressortissants de sa patrie d'adoption, la musicologie ne l'occupera pratiquement plus. Nous n'avons ainsi aucune correspondance suivie importante de Rolland avec un musicologue ou même un musicien suisse, sinon avec Arthur Honegger et Ernest Bloch ${ }^{4}$, qui étaient des expatriés. Nous savons certes que c'est au Zurichois Ernst Kurth que l'auteur de Jean-Christophe doit, en 1925, la découverte, tardive, de Bruckner ${ }^{5}$; et le fait qu'il publie en 1936 dans la revue Schweizerische Instrumentalmusik une manière de bref testament musical ${ }^{6}$ est assurément significatif, mais ces éléments restent insuffisants à documenter des relations vraiment serrées entre Rolland et le milieu musical suisse.

Nous préférerons donc nous intéresser ici à un aspect très particulier des relations de Rolland avec la vie artistique suisse, à savoir son témoignage sur des formes théâtrales dont la spécificité helvétique l'a d'autant

2. Il évoque dans ses Mémoires et fragments du Journal, p. 27, "l'émotion de [sa] rencontre avec le vieux barde, à Villeneuve, en Suisse, le 19 août 1883».

3. Lettre du 8 septembre 1938, in A. Blum (éd.), Correspondance entre Romain Rolland et Charles Baudouin, p. 227.

4. Voir J.-F. Tappy (éd.), Ernest Bloch, Romain Rolland.

5. Voir A. Corbellari, Les mots sous les notes, p. 232.

6. "La lumière du soleil", article reproduit dans A. Corbellari, Les mots sous les notes, p. 333 sq. 
plus frappé qu'il cherchait lui-même depuis son adolescence à renouveler le théâtre français.

Rien de moins original, au demeurant, qu'un tel désir: à la fin du XIX siècle, il n'est presque pas un écrivain français qui ne le fasse sien. Et de fait, d'Antoine, avec son Théâtre-libre (1887), à Copeau, avec le Vieux-Colombier (1913), en passant par Lugné-Poe et le Théâtre de l'CEuvre (1893), auquel Rolland fut associé, puisqu'il fit jouer deux de ses pièces par cette troupe (Aërt et Les Loups, toutes deux en 1898), on assiste effectivement à un extraordinaire renouvellement de l'horizon du théâtre français qui, avec Claudel, Giraudoux, Montherlant, Anouilh et bien d'autres s'épanouira véritablement à partir de l'entre-deux-guerres.

La tentative dont Rolland a été le plus proche reste celle de Maurice Pottecher qui ouvrit en 1895 un théâtre en plein air à Bussang, village des Vosges alors situé à la frontière de la France et l'Alsace allemande. Ce "Théâtre du peuple» donna des représentations jusqu'en 1911 et Rolland s'attacha à l'entreprise au point d'y passer une bonne partie de l'été 1897. Pottecher et Rolland écriront ainsi chacun, dans les mêmes années, un ouvrage intitulé Le Théâtre du peuple. Celui de Rolland, publié en 1903, revu en 1913 et récemment réédité, est à la fois apologétique, glorifiant la démarche de Pottecher, et prospectif, dessinant les bases d'une conception qui ne trouvera, il faut bien le dire, qu'assez peu d'occasions de se concrétiser dans l'œuvre même de Rolland, lequel ne se consacrera en effet plus que très occasionnellement au théâtre après 1903: la farce pacifiste de Liluli (1915), soutenue en 1922 par une musique de Honegger, et surtout les grandes représentations de la pièce à grand spectacle $L e$ 14 juillet du 14 au 23 juillet 1936, au lendemain de la victoire du Front populaire (avec rideau de scène de Picasso!), eurent finalement peu de répercussions ${ }^{7}$ et Chantal Meyer-Plantureux n'a malheureusement pas tort de constater qu' "aujourd'hui le nom de Romain Rolland et son rôle dans l'élaboration d'un théâtre nouveau sont complètement tombés dans l'oubli" 8 .

Mais si Romain Rolland repousse dans le futur la réalisation d'un tel théâtre, il ne se fait pas faute d'oublier d'évoquer ses précurseurs, en

7. Un collectif de sept musiciens (Roussel, Ibert, Milhaud, Honegger, Koechlin, Auric et Lazarus), parmi lesquels on trouve quelques-uns des compositeurs français les plus en vue de l'époque, se partageait la musique de scène.

8. Ch. Meyer-Plantureux, "Préface», in R. Rolland, Le Théâtre du peuple, p. 17. 
particulier Rousseau qui voyait déjà le modèle d'une telle entreprise dans les fêtes suisses, et auquel Rolland se réfère évidemment longuement. Dans Le Théâtre du peuple, il rappelle ainsi la propension des Suisses, "soutenus par l'orgueil et l'amour de la petite patrie» pour le théâtre populaire, "dont la tradition n’a jamais été interrompue en Suisse depuis des siècles" ". Il remonte ainsi jusqu'à un Guillaume Tell joué à Zurich en 1545 et cite l'ouvrage de Stocker, Das Volkstheater in der Schweiz (1893), avant de recenser un nombre impressionnant de spectacles suisses créés dans les années précédentes ${ }^{10}$, sans oublier bien sûr la fameuse Fête des Vignerons de Vevey, dont il estime cependant que "jusqu'à ces dernières années n'étaient sorties de ce mouvement dramatique que peu d'œuvres populaires, dignes de survivre à l'occasion qui les avait fait naître» ${ }^{11}$.

Mais ces réticences n'empêchaient pas Rolland d'attendre ce spectacle avec impatience. Ainsi le voit-on faire part à Richard Strauss de son désir de se rendre à la Fête des Vignerons de 1905, lui avouant craindre de ne pas avoir l'occasion d'assister à la prochaine ${ }^{12}$, ce en quoi il se montrait trop pessimiste, puisqu'en 1927 il fut d'autant mieux placé pour assister à l'édition suivante de la manifestation (dont la musique avait été confiée, pour la seconde fois consécutive, à Gustave Doret) qu'il habitait depuis cinq ans à Villeneuve.

Le Théâtre du peuple restant avare de commentaires sur les manifestations théâtrales évoquées, c'est vers la correspondance et d'autres écrits de circonstances de Romain Rolland que l'on se tournera pour lire des considérations plus détaillées, et souvent riches d'ambivalences significatives, sur les spectacles vus en Suisse par notre auteur. La Fête des vignerons de 1905 présente ainsi, dans le compte rendu qu'il en fait à sa confidente Sofia Bertolini, des relents à la fois nietzschéens et rousseauistes:

J'ai été à la Fête des Vignerons de Vevey, la semaine passée. C'était vraiment un spectacle unique. Un amphithéâtre en plein air de 20.000 personnes, 2 à 3000 acteurs; un soleil de feu; et des jeux qui faisaient penser à ceux de la Grèce antique. Le cadre était celui des Quatre

9. Voir R. Rolland, Le Théatre du peuple, p. 171, 174-176 et 178 sq. (n. 49, 68, 70, 71 et 91).

Io. Dans l'édition revue de 1913, la liste va jusqu'en 1911.

II. R. Rolland, Le Théâtre du peuple, p. 178.

I2. Richard Strauss et Romain Rolland, p. 49 (lettre du 3 août 1905). 
Saisons. C'étaient des paysans, bouviers, filles des champs, qui étaient acteurs, chanteurs et danseurs; et cela ne manquait ni de grandeur, ni même de grâce et de beauté ${ }^{13}$.

On voit ici un Rolland encore pris par l'enthousiasme de la représentation: il est clair que les réticences exprimées en 1913 dans la réédition du Théâtre du peuple témoignent d'une relativisation et d'une prise de distance a posteriori.

Notons en passant que l'on rechercherait en vain chez Romain Rolland des allusions à la ferveur religieuse que véhicule souvent ce théâtre en plein air. Trois quarts de siècle avant lui, Rodolphe Töpffer relatait dans son Voyage autour du Mont-Blanc, une représentation populaire en plein air dans la ville suisse allemande de Stalden en des termes dont l'imprégnation rousseauiste n'est pas douteuse, mais où domine encore le sentiment de la catharsis morale opérée par ce type de spectacle:

Que si nous résumons maintenant l'effet que doit produire sur les hommes auxquels elle est destinée une représentation comme celle qui a eu lieu à Stalden, nous n'hésitons pas à croire, qu'à la condition qu'une pareille représentation ne se répète qu'à de très longs intervalles, elle ne peut que produire l'effet moral le plus salutaire et le plus durable. Ceci frappe, touche, élève également les âmes de ces montagnards. Au sortir d'une fête pareille, ils s'en retournent sur leurs rochers approvisionnés d'impressions saines, fortes, pieuses; de tableaux et d'exemples dont longtemps la tradition va remplir avec avantage leurs entretiens et amuser utilement leurs veillées. Ils n'ont entrevu du beau que son côté sérieux, de l'art que ses applications respectables, de la scène que son éloquente moralité ${ }^{14}$.

Le genre s'est visiblement laïcisé aux yeux d'un Romain Rolland, dont la première expérience théâtrale suisse remonte vraisemblablement à 1899: il assista en effet cette année-là à Altdorf à une représentation en plein air du Guillaume Tell de Schiller, dont il rendit compte dans le Journal des Débats ${ }^{15}$; le spectacle, dans l'ensemble, l'avait convaincu, mais il

I3. Chère Sofia, t. I, p. 231 (lettre du 13 août 1905).

I4. R. Töpffer, "Voyage autour du Mont Blanc", in Nouveaux voyages en zig-zag, p. 265 sq.

15. Cité par B. Duchatelet, Romain Rolland tel qu'en lui-même, p. 97. 
regrettait que le décor ait été réalisé en dépit du bon sens: le théâtre était clos et l'on avait dépensé des trésors d'habileté pour brosser une toile de fond représentant le lac, alors qu'il aurait suffi d'ouvrir le théâtre pour avoir l'original du même paysage sous les yeux!

Romain Rolland assiste aussi, en 1903, sur les hauts de Lausanne, à un grand spectacle rassemblant 2500 participants, apparemment très proche par l'esprit de la Fête des Vignerons, mais qui lui a cette fois-ci plutôt déplu; il critique en particulier la musique d'Emile Jaques-Dalcroze, profitant d'épingler au passage la médiocrité des compositeurs suisses d'alors: «le compositeur, Jaques-Dalcroze, est un des deux ou trois musiciens suisses les meilleurs; mais ce n'est pas beaucoup dire ${ }^{16}$ ", lâche-t-il à sa correspondante. La musique de Doret qu'il appréciera à Vevey en 1905 sera pourtant de la même veine. Mais ce qui semble le gêner particulièrement dans le spectacle lausannois, qu'il juge «extraordinaire et absurde", c'est son aspect hybride; estimant que "rien n'était plus faux que ce grand Opéra en plein air, joué par un vrai peuple», il dénonce une confusion des genres: la pièce est "entièrement en musique", ce qui est contraire à l'idée que Rolland se fait d'un type de spectacle qui doit jouer sur l'alternance des types dramatiques et non sur la prédominance d'un seul. Or, le chœur, qui aurait pu être sublime dans un cadre plus naturel, est omniprésent, et lui paraît donc jouer à contre-emploi. Au surplus, Jaques-Dalcroze n'a, selon lui, tout simplement pas les moyens de son ambition. La surenchère musicale n'est justifiable, chez Wagner, que par le génie, et le compositeur genevois, aux yeux de Romain Rolland, n'en a aucun.

Dans Le Théâtre du peuple, en revanche, Rolland s'abstient de critiquer le spectacle de Jaques-Dalcroze, tout en en tirant, à propos de la musique qui doit habiller un tel spectacle, des considérations générales qui ne manquent pas d'ambiguïté:

La musique est très utile, - au second plan. - Elle doit être le fond de la fresque, le support de l'action, l'atmosphère du drame. Il faut qu'elle imprègne chaque scène du coloris qui lui convient, et qu'elle n'attire jamais l'attention à elle, qu'elle se sacrifie au drame. Il faut, en un mot, qu'elle soit à la fois intelligente et désintéressée. (Mais je demande l'impossible.) ${ }^{17}$

I6. Chère Sofia, t. I, p. 123 (lettre du 7 juillet 1903).

17. Le Théâtre du peuple, p. 114. 
L'intéressant est que la difficulté, voire la contradiction, que décrit Rolland ne reste pas pour lui pur objet de discours. Dans la pièce clé du "Théâtre de la Révolution", Le Quatorze Juillet, qu'il vient juste d'écrire, en 1903, et dont son essai est le manifeste implicite, la question de l'accompagnement musical se pose très précisément. Par ses dimensions et par le nombre considérable de participants qu'elle requiert, cette pièce est la plus ambitieuse du cycle révolutionnaire de Rolland. On sait que, pour la création, en 1902, il se contenta de vingt acteurs et de quarante figurants ${ }^{18}$; il évoque cependant, en décembre 1906, la possibilité de faire rejouer la pièce le 14 juillet de l'année suivante sur la place de l'Hôtel-de-Ville, à Paris, "avec 1500 ou 2000 acteurs " ${ }^{19}$, donc avec un effectif comparable à celui d'une Fête des Vignerons. Le projet tombera à l'eau, mais les représentations de 1936 offriront à notre dramaturge, on l'a rappelé, une éclatante quoique tardive revanche.

La sévérité de Romain Rolland envers les compositeurs de son temps (et même avec ceux du passé), est bien connue ${ }^{20}$, et l'on ne doit donc pas s'étonner que les musiciens suisses ne trouvent guère grâce à ses yeux. Au demeurant, Doret et Jaques-Dalcroze ${ }^{21}$ sont tous deux des représentants de ce que l'on pourrait nommer cum grano salis «l'école nationale» suisse romande: ils s'inspirent de l'art populaire de leur terroir, cherchant à le revitaliser, et l'un n'a pas plus que l'autre la prétention de rivaliser avec Wagner, comme Rolland semble en accuser le second («Et que n'aurait pu faire un Wagner avec de tels moyens!» ${ }^{22}$ ). Une telle opinion semble suffire à expliquer que les relations de Rolland avec les compositeurs suisses n'aient jamais été fort étroites, mais on peut regretter que notre musicologue n'ait guère été sensible à la dimension rythmique et chorégraphique qui fait l'originalité essentielle de l'œuvre de Jaques-Dalcroze;

I8. Chère Sofia, p. 60 (lettre du 8 mars 1902).

19. Ibid., p. 279 (lettre du $1^{\text {er }}$ décembre 1906).

20. Même Richard Strauss, qui est pour lui son contemporain capital dans le domaine de la composition, n'échappe pas à des critiques parfois acerbes. En revanche, Rolland, poussé par son désir de découvrir les compositeurs de l'avenir fut aussi d'une surprenante indulgence avec des auteurs aujourd'hui bien oubliés comme Lorenzo Perosi ou Paul Dupin (voir A. Corbellari, Les mots sous les notes, p. 205-209 et 217-223).

2I. Sur ce dernier, voir l'excellente petite biographie d'A. Berchtold, Emile JaquesDalcroze et son temps. Pour une vue d'ensemble plus large, on consultera également, du même auteur, La Suisse romande au cap $d u X X^{e}$ siècle.

22. Chère Sofia, t. I, p. 123. 
s'il avait réellement cherché à savoir ce qui se cachait derrière les apparences, l'auteur du Théâtre du peuple aurait pu comprendre que ce petit homme courtaud était tout simplement en train de révolutionner le rapport des musiciens à leur propre corporalité. Il est vrai que l'on touche là à l'un des points aveugles de l'esthétique de Rolland: autant, en effet, celui-ci, cherche à se dégager de l'intellectualisme desséchant, autant il reste, paradoxalement, sourd à l'injonction de Nietzsche réclamant du philosophe qu'il sache danser ${ }^{23}$.

En fin de compte, le seul créateur suisse qui lui a semblé sortir quelque peu du lot, en tant que talent individuel, est le dramaturge René Morax, dont Rolland créditait les «drames vaudois d'un vigoureux et tranquille sentiment populaire » ${ }^{24}$, et dont le Théâtre du Jorat, inauguré le 9 mai 1908 à Mézières, en plein canton de Vaud, fut, on le sait, une entreprise en tous points comparable à celle de Bussang ${ }^{25}$. Pierre Meylan cite d'ailleurs une intéressante lettre de Rolland à Morax où l'auteur des Loups dit son incompréhension devant les tentatives de ressusciter le chœur antique dans le théâtre populaire moderne:

Je ne m'explique pas cette survivance du passé dans la pensée moderne: cela me paraît un poids mort. Le chœur antique a été particulièrement, je crois, quelque chose de local et accidentel. Il suppose des conditions sociales, religieuses, musicales, plastiques, qui n'ont pas duré plus d'un siècle ou deux. Je crains l'idéal antique. Il ne me semble plus répondre aux besoins d'aujourd'hui; et je redoute qu'il ne mette une barrière de plus entre la pensée de l'élite et le peuple, qui en est déjà bien assez loin. Mais peut-être que je me trompe ${ }^{26}$.

23. On lira, à cet égard, avec intérêt les pages de Ph. Grosos (L'existence musicale, p. 7-95) sur le lien nécessaire (et trop souvent occulté par les commentateurs du philosophe allemand) tissé par Nietzsche entre musique et danse.

24. R. Rolland, Le Théâtre du peuple, p. 132.

25. Mentionnons encore tout de même une pièce vue par Rolland à Morschach au-dessus du lac des Quatre-Cantons en 1911, sur Marignan: "la pièce était une des meilleures qu'on ait écrites en Suisse, vivante, bien observée, pleine de types locaux, et d'une langue savoureuse" (lettre du 15 septembre 1911 à Alphonse de Châteaubriant, in L'un et l'autre, p. 103).

26. Lettre du 17 juin 1906, citée dans P. Meylan, Arthur Honegger et René Morax au Théâtre du Jorat, p. 14. 
Volontiers tenté par le vitalisme nietzschéen (qu'il saluera autant chez Richard Strauss que chez D’Annunzio!), Rolland semble prendre ici ses distances à la fois envers l'auteur de La Naissance de la tragédie et envers Wagner: on se souviendra qu'il a d'ailleurs été le premier, comme le rappelle André Couroy, à ironiser sur le culte de la personnalité dont Wagner était l'objet à Bayreuth ${ }^{27}$.

Evoquant la musique de scène du 14 Juillet dans la préface de son Théâtre de la révolution, Rolland tiendra pourtant des propos très éloignés des considérations que l'expérience des fêtes suisses lui avait suggérées :

Il faut, pour donner à l'œuvre son couronnement logique et au fait historique sa portée universelle, l'entrée en scène d'une puissance nouvelle: la Musique, la force tyrannique des sons, qui remue les foules passives; cette illusion magique, qui supprime le Temps, et donne à ce qu'elle touche un caractère absolu.

La musique doit être ici le fond de la fresque, la trame des paroles. Pas un instant elle ne doit se taire, - tantôt forte et distincte, tantôt douce et voilée. Son office est de préciser le sens héroïque de la fête, et de combler les silences qu'une foule de théâtre ne peut jamais réussir à remplir complètement, qui s'ouvrent malgré tout au milieu de ses cris, et qui détruisent l'illusion de la vie continue. Il n'est pas nécessaire que le public saisisse tous les mots de la foule, pas plus que toutes les notes de l'orchestre et des chœurs; il faut qu'il ait seulement l'impression d'une kermesse triomphante ${ }^{28}$.

On ne peut qu'être frappé par le ton très wagnérien de ces considérations: l'«illusion magique», le "caractère absolu », l'idée de supprimer le temps, la volonté de créer une trame musicale ininterrompue et de relayer un chœur impuissant à créer à lui seul "l'illusion de la vie continue», enfin cette apothéose du dionysiaque, qui est une constante de la vision populaire de Rolland ${ }^{29}$ : autant d'expressions et de vœux qui s'appliquent presque davantage au drame de Wagner qu'à celui que l'auteur de JeanChristophe tente de créer, car ce qu'il affirme être l'âme de ce théâtre - le Peuple - se trouve paradoxalement relégué au second plan, alors même

27. Voir A. Coeuroy, Wagner et l'esprit romantique, p. 318 sq.

28. Le Théâtre de la Révolution, p. 150.

29. Que l'on songe à son roman Colas Breugnon. 
que c'était bien la musique qui devait, dans l'utopie du Théâtre du peuple, occuper cette place subalterne.

On comprend par ailleurs que la description de Rolland ait tenté les musiciens: dès 1911, Albert Doyen écrivait, pour la scène finale du Quatorze Juillet, une musique sous-titrée Le Triomphe de la Liberté, que Rolland trouvait "d'un excellent style populaire" ${ }^{30}$, mais qui reste cependant sans commune mesure avec la création collective de 1936, au sein de laquelle la présence de Honegger peut sembler particulièrement emblématique, puisque le compositeur du Roi David a été le plus célèbre collaborateur de Morax au Théâtre du Jorat. Une lettre de Rolland à Honegger, à propos de leur collaboration de 1922 sur Liluli, nous est, d'ailleurs, un témoignage précieux des intentions musicales de Rolland. Pas plus que dans le cas du Quatorze Juillet, l'auteur de Jean-Christophe ne s'embarrasse de détails techniques. Ainsi désire-t-il, à un moment donné, que la scène soit "enveloppée délicatement de musique enjôleuse et enivrée»; ailleurs, il veut une "marche burlesque», et le "chant évocateur" de l'héroïne doit avoir "des caractères nouveaux, incisifs, agressifs, impérieux, des trilles suraigus " ${ }^{31}$. Ces exigences étonnent par leur modestie; il est vrai qu'Honegger n'avait à sa disposition qu'une flûte, un violoncelle, un piano, une soprano et un chœur à deux voix. Mais la minceur du propos semble aussi un choix de Rolland qui n'hésite pas, ici, à faire exprimer par la musique non tant la sublimité de son idéal que le grotesque d'un monde corrompu. La vraie musique est ailleurs, dans Liluli, inaccessible peut-être, comme le dieu caché des gnostiques, ou la musique des sphères médiévales: seule sa caricature est audible et, comme telle, ne saurait représenter que la face négative d'un désir éperdu de communion par l'art.

En même temps, on peut voir dans ce minimalisme un hommage à l'esprit du "théâtre du peuple» et un retour à la simplicité des traditions helvétiques qu'Honegger a su magnifier dans ses musiques destinées au théâtre de Mézières.

Ainsi, au-delà de ses rêves de grandeur, Rolland, décidément ballotté par ses ambivalences, retrouve-t-il cette simplicité rousseauiste et, peut-

30. Lettre inédite du 29 décembre 1918 à André Pirro (Bibliothèque nationale de France, fonds Romain Rolland).

3I. Lettre du 18 octobre 1922, de Arthur Honegger, citée dans M. Reinhardt, «Romain Rolland conseille Honegger», p. 144. 
être, antique prônée dans son essai. Au bout du compte, la communion des spectateurs à travers la présentation épurée d'un message de fraternité l'emporte sur la communication mystique des âmes, et la perfection artistique cède la première place à une sincérité qui assure la pérennité d'une catharsis libératoire. Parti de l'idéal des spectacles populaires de la Suisse traditionnelle, Rolland projette dans l'avenir un théâtre peut-être encore à inventer.

Alain CORBELLARI

Universités de Lausanne et de Neuchâtel 


\section{BIBLIOGRAPHIE}

\section{Sources}

Blum, Antoinette (éd.), Correspondance entre Romain Rolland et Charles Baudouin: une si fidèle amitié: choix de lettres (1916-1944), avantpropos d'Yves Baudouin, Meyzieu, Césura, 2000.

Chère Sofia: choix de lettres de Romain Rolland à Sofia Bertolini GuerrieriGonzaga, 2 vols, Paris, Albin Michel, 1959-1960 (Cahiers Romain Rolland 10 et 11 ).

L'un et l'autre: correspondance entre Romain Rolland et Alphonse de Châteaubriant: choix de lettres 1906-1914, préface de L.-A. Maugendre, Paris, Albin Michel, 1983 (Cahiers Romain Rolland 26).

Richard Strauss et Romain Rolland: correspondance, fragments de journal, Paris, Albin Michel, 1951 (Cahiers Romain Rolland 3).

Romain, Rolland, Le Théâtre du peuple, Paris, Cahiers de la Quinzaine, 1903 ; rééd. Paris, Albin Michel, 1926; rééd. de Chantal MeyerPlantureux, Bruxelles, Complexes, 2003.

—, Le Théatre de la Révolution, Paris, Hachette, 1909.

—, Colas Breugnon, Paris, Ollendorff, 1918; rééd. Le Livre de Poche, 1986.

—, Mémoires et fragments du Journal, Paris, Albin Michel, 1956.

Tappy, José-Flore (éd.), Ernest Bloch, Romain Rolland: lettres: 1911-1933, Lausanne, Payot, 1984.

\section{Travaux}

Berchtold, Alfred, La Suisse romande au cap du XX $X^{e}$ siècle. Portrait littéraire et moral, Lausanne, Payot, 1964. 
-, Emile Jaques-Dalcroze et son temps, Lausanne, L'Age d'Homme, 2000.

Coeuroy, André, Wagner et l'esprit romantique, Paris, Gallimard, 1964. Corbellari, Alain, Les mots sous les notes. Musicologie littéraire et poétique musicale dans l'auvre de Romain Rolland, Genève, Droz, 2010.

Duchatelet, Bernard, Romain Rolland tel quien lui-même, Paris, Albin Michel, 2002.

Grosos, Philippe, L'existence musicale. Essai d'anthropologie phénoménologique, Lausanne, L'Age d'Homme, 2008.

Meyer-Plantureux, Chantal, Romain Rolland - théatre et engagement, Caen, Presses universitaires de Caen, 2012.

Meylan, Pierre, Arthur Honegger et René Morax au Théatre du Jorat, Lausanne, Editions du Cervin, 1965.

Reinhardt, Marc, «Romain Rolland conseille Honegger», Revue musicale suisse, 3 (1969), p. 144-146.

Töpffer, Rodolphe, Nouveaux voyages en zig-zag, Paris, Lecou, 1853. 
